\title{
EDITORIAL
}

\section{Superficial tumors of the gastrointestinal mucosa: the problem of the histological diagnosis of intravascular infiltration in tissues from endoscopic submucosal dissection}

Alfredo J. A. Barbosa

The current progress in gastrointestinal endoscopy has shed some light on the medical practice related to the semiology and treatment of tumors of the digestive tract, thus resulting in a high commitment to early diagnosis and the ensuing effective treatment of these neoplasms. Within this perspective, it is clear today that the relative frequency of advanced gastrointestinal tumors decreased dramatically due to the increased number of diagnosis of early neoplastic lesions. The great advantage of this event is the fact that these tumors are likely to be treated permanently and without major mutilations. Moreover, progress in endoscopic techniques does not seem to show signs of saturation. Instead, there are increasingly more technological possibilities that provide a thorough examination of the various segments of the digestive tract, comprising magnification and image resolution techniques that allow a reliable exam of the main distinguishing features of the gastrointestinal mucosa. New endoscopic approaches are emerging, among which the high-resolution confocal laser endomicroscopy is particularly worth mentioning, which evaluates in vivo morphological changes of tissues present in the superficial layers of the mucosa ${ }^{(5)}$.

Although the accuracy of morphological findings has advanced due to endoscopic procedures applied to analyze gastrointestinal mucosal lesions, histopathological examination has been a mandatory complement in most cases. Therefore, the suspected neoplastic lesion identified by endoscopy always seems to be dependent on confirmation by histopathological results. Accordingly, histopathology has always followed closely the rapid development of endoscopic techniques. Once the lesion is detected and the nature of the tumor is confirmed, though still limited to the mucosa and superficial submucosa regions, its removal is required, either by conventional or laparoscopic surgical intervention, or by means of endoscopic procedures.

Endoscopic surgery for removal of superficial tumors of the gastrointestinal mucosa, still in the initial phase of implementation, has become progressively more plausible. It consists mostly of two surgical methods that resulted from Japanese researchers' work: Endoscopic Mucosal Resection (EMR) and Endoscopic Submucosal Dissection (ESD). As it was initially proposed, EMR consists of a minimally invasive technique used for diagnosis, staging and treatment of superficial tumors, including the sessile polyps of the gastrointestinal mucosa ${ }^{(7)}$. In contrast, ESD results in relatively large en bloc resections, allowing lower rates of local tumor recurrence and better pathological assessment ${ }^{(2,8)}$. Although these surgical procedures have emerged as important therapeutic modalities for superficial tumors of the gastrointestinal mucosa, they are not widespread yet, inasmuch as they are highly dependant on the endoscopist's expertise. It appears that we are entering a new era in the chapter of gastrointestinal surgery. In brief, we can mention three bases that ensure a promising future for endoscopic surgery: (1) en block resection of the lesion, allowing the complete histopathological analysis of the tumor, including study of surgical safety margins in several sites; (2) better quality of life for the patient because of the low rate of morbidity compared to conventional surgical procedures; (3) complete cure for all patients, or at least most of them, in non-metastatic cases.

Professor of Pathology at Universidade Federal de Minas Gerais-Medical School (UFMG-MS); associate editor of the Brazilian Journal of Pathology and Laboratory Medicine (Jornal Brasileiro de Patologia e Medicina Laboratorial [JBPML]). 
It is known that the presence of lymph node metastasis of superficial neoplasms of the digestive mucosa is an uncommon possibility, especially considering tumors restricted to the mucosa, which occurs in less than $3 \%$ of them, according to the Japanese research $^{(9)}$. Accordingly, the question regarding the presence of metastases is frequently relegated to the sidelines when endoscopic resection of these tumors is programmed. Therefore, the question remains without a definite answer. A thorough examination of the endoscopically resected superficial tumor does not contribute significantly to clarify this issue. The presence of surgical safety margins at all levels of resection does not help much in this regard, although it is a parameter that must be taken into account in the postoperative period. One possible strategy that has been deployed by researchers in the field of pathology is the attempt to identify the presence of vascular infiltration by neoplastic cells in the surgical specimen. Although lymphovascular invasion does not ensure the existence of metastases, the question accordingly acquires more consistency and the consensus on medical follow-up may include the participation of the oncologist in order to administer adjuvant treatment, chemotherapy or radiotherapy.

Therefore, the search for lymphovascular invasion in endoscopic resection of superficial tumors of the gastrointestinal mucosa becomes an auxiliary tool, hence improving postoperative patient follow-up. Nevertheless, despite the seemingly simple task of looking for vascular infiltration, pathologists may find pitfalls that may lead to inaccurate diagnosis. It is particularly worth noting that, among other reasons, the frequent interference of subjectivity in the interpretation of these lesions is not negligible ${ }^{(6)}$. This accounts for the fact that the recognition of morphological characteristics of lymphovascular invasion in histological sections routinely stained with hematoxylin and eosin (HE), or even with special stains, is subject to controversy. Besides the variability of interpretation by different observers, other widely known factors may affect the impartiality of the investigation into the presence of vascular invasion. Some of them may be mentioned: (a) the accurate diagnosis is often dependent on the number of histological sections, which varies in different studies; (b) tissue retraction artifacts caused by the use of formaldehyde as a fixative may cause images simulating vascular spaces; (c) stromal fibroblasts may simulate endothelial cells; (d) artifacts produced by the blade during microtomy of paraffin blocks may raise some doubts about voids in tissue with neoplastic elements.

Contributing to a more accurate diagnosis of neoplastic infiltration of small vessels, the use of immunohistochemical staining with vascular endothelium markers has been proposed. By using this method, some authors have reported more accurate results in the identification of small blood and lymphatic vessels presenting neoplastic cell infiltration ${ }^{(4,10)}$. The use of immunohistochemistry to identify micrometastases of gastrointestinal tumors is not recent. This approach has contributed significantly to more accurate diagnoses in cases related to the identification of small neoplastic lesions since the early $90 \mathrm{~s}^{(3)}$.

Therefore, the importance of histopathological diagnosis of vascular infiltration in surgically removed tissues must be acknowledged, particularly in cases of superficial tumors. This may exert considerable influence on the medical procedure during the postoperative period. In this issue of the Brazilian Journal of Pathology and Laboratory Medicine (JBPML), Alderman et al. present the results of their research into lymphovascular infiltration in 30 cases of ESD due to superficial cancer in different segments of the digestive tube ${ }^{(1)}$. The authors compared the histopathological findings from HE stained preparations with those obtained through immunohistochemistry, in which antibodies against vascular endothelium were deployed. The results presented by the authors add further information as to the anatomical and clinical staging of patients with superficial tumors submitted to ESD. The issue on the possibility of metastases in these patients remains and, in principle, pathologists will not solve it at first place, insofar as this exceeds the limits of their competence.

\section{REFERENCES}

1. ALDEMAN, N. L. S. et al. The role of immunohistochemistry in the detection of vascular invasion in specimens of endoscopic submucosal dissection. J Bras Patol Med Lab, v. 49, n. 4, p. 273-77, 2013.

2. CHAVES, D. A. et al. Initial experience of endoscopic submucosal dissection in Brazil to treat early gastric and esophageal cancer: a multi-institutional analysis. Arq Gastroenterol, v. 50, n. 2, p. 148-52, 2013. 
3. CUTAIT, R. et al. Restaging of colorectal-cancer based on the identification of lymph-node micrometastases through immunoperoxidase staining of CEA and cytokeratins. Dis Colon \& Rectum, v. 34, n. 10, p. 917-20, 1991.

4. DU, J. R. et al. Vascular endothelial growth factor and microvascular density in esophageal and gastric carcinomas. World J Gastroenterol, v. 9, n. 7, p. 1604-6, 2003.

5. GOETZ, M.; KIESSLICH, R. Advances of endomicroscopy for gastrointestinal physiology and diseases. Am J Physiol Gastrointest Liver Physiol, v. 298, n. 6, p. G797-806, 2010.

6. HARRIS, E. I. et al. Lymphovascular invasion in colorectal cancer: an interobserver variability study. Am J Surg Pathol, v. 32, n. 12, p. 1816-21, 2008.

7. INOUE, H. et al. Endoscopic mucosal resection with a cap-fitted panendoscope for esophagus, stomach, and colon mucosal lesions. Gastrointest Endosc, v. 39, p. 58-62, 1993.

8. ISHIHARA, R. et al. Comparison of EMR and endoscopic submucosal dissection for en bloc resection of early esophageal cancers in Japan. Gastrointest Endosc, v. 68, p. 1066-72, 2008.

9. KOJIMA, T. et al. Outcome of endoscopic mucosal resection for early gastric cancer: review of the Japanese literature. Gastrointest Endoscop, v. 48, p. 550-4, 1998.

10. PRADEEP, C. R.; SUNILA, E. S.; KUTTAN, G. Expression of vascular endothelial growth factor (VEGF) and VEGF receptors in tumor angiogenesis and malignancies. Integr Cancer Ther, v. 4, n. 4, p. 315-21, 2005. 